\title{
Eine geometrische Interpretation der Ausgleichsrechnung
}

\author{
ANDreas ASPERL
}

Abstract. Using real examples of applied mathematics in upper secondary school one has do deal with inaccurate measures. This will lead to over constrained systems of linear equations. This paper shows an instructive approach which uses methods of descriptive and computer aided geometry to get a deeper insight into the area of calculus of observations. Using a qualified interpretation one can solve problems of calculus of observations with elementary construction techniques of descriptive geometry, independent of the norm one uses.

Key words and phrases: Ausgleichsrechnung, Darstellende Geometrie, computergestützte Geometrie.

ZDM Subject Classification: G04, G84, R2, N54.

\section{Motivation}

In manchen Unterrichtssequenzen kann es vorkommen, dass gegebene Angaben eines Objekts dieses über bestimmen; so kann es bereits im Grundkurs Mathematik passieren, dass beim Abmessen der Dimensionen eines Raumes gewisse Maße einander widersprechen (Abbildung 1).

Bemerkung. Zur einfacheren Ausführung der Zeichnung werden eher unrealistische Messwerte angenommen; die Schüler sollen das Prinzip der Ausgleichsrechnung und damit zusammenhängende Probleme erkennen. 


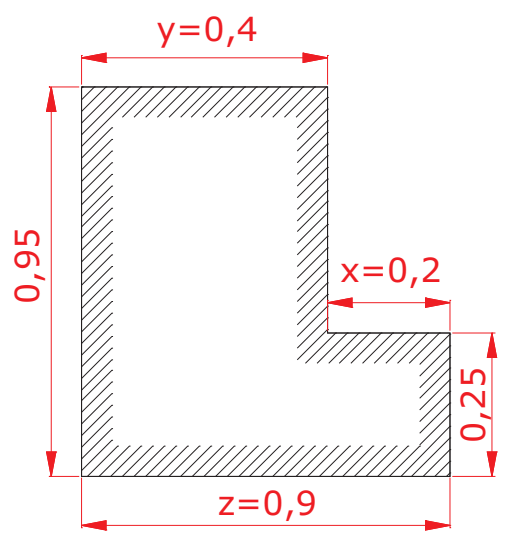

Abbildung 1

Unter der Voraussetzung, dass sämtliche Seitenwände des Wohnraumes normal zueinander stehen, gab es offensichtlich beim Bestimmen der „horizontalen“ Dimensionen Messungenauigkeiten, da $0,4+0,2=0,6<0,9$ gilt. Wie sollen die Werte für $x, y$ und $z$ nun tatsächlich gewählt werden, so dass die auftretenden Messfehler möglichst gering sind, und was bedeutet „möglichst geringe Messfehler"?

Bemerkung. In der Praxis sollte man, um Beobachtungs- bzw. Messfehler möglichst auszugleichen, Messungen mehrfach und von verschiedenen Personen mit verschiedenen Messgeräten ausführen lassen.

\section{Mathematische Fassung}

Zur Beantwortung der offenen Fragen, fassen wir die drei gemessenen Resultate vorerst mathematisch als lineares Gleichungssystem in den Variablen $x$ und $y$.

So erhalten wir mit

$$
\begin{aligned}
x & =0,2 \\
y & =0,4 \\
x+y & =0,9
\end{aligned}
$$


ein überbestimmtes lineares Gleichungssystem, welches nur für exakt gemessene Werte lösbar ist. Da keine exakten Messungen vorliegen, dürfen wir aber auch nicht erwarten, dass die gesuchten Größen $x$ und $y$ die Gleichungen (1) erfüllen.

Bezeichnen wir nun die bei den drei Messvorgängen auftretenden Messfehler mit $r_{1}, r_{2}$ und $r_{3}$ und berücksichtigen wir diese in unserem Ansatz, so erhalten wir das Gleichungssystem

$$
\begin{aligned}
x-0,2 & =r_{1} \\
y-0,4 & =r_{2} \\
x+y-0,9 & =r_{3}
\end{aligned}
$$

Bemerkung. Die Gleichungen (2) werden als Fehlergleichungen, die Größen als Residuen bezeichnet. [2]

Im Gleichungssystem (2) sind die zu den exakten Größen $x$ und $y$ gehörigen Residuen $r_{i}$ nicht bekannt; wir wissen nur, dass das System nicht mit $r_{1}=r_{2}=$ $r_{3}=0$ erfüllbar ist. Eine sinnvolle Aufgabenstellung ist es nun, zu fragen, ob es zwei Zahlen $x_{0}$ und $y_{0}$ gibt, die eingesetzt in die Fehlergleichungen (2) Residuen ergeben, die minimal sind. Wobei wir noch nicht festgelegt haben, was minimal in diesem Zusammenhang bedeuten soll.

Folgende Festlegungen für minimale Residuen wären denkbar (und führen auf mathematisch sinnvolle Fragestellungen):

a) die Summe der Beträge der Residuen sei minimal

b) der Betrag des größten auftretenden Messfehlers sei minimal

c) die Summe der Quadrate der Messfehler sei minimal.

Bemerkung. Wir verwenden jeweils nur die Beträge der Messfehler, da sonst der Fall eintreten könnte, dass gleich große, entgegengesetzt orientierte Messfehler einander aufheben.

Wir wollen mit der Fragestellung c) beginnen, da dies auf ein einfach durchführbares, und daher gebräuchliches Rechenverfahren (Methode von Gauss: Summe der kleinsten Fehlerquadrate) führt:

Bemerkung. Der geometrische Zugang ermöglicht uns auch die Lösung der beiden anderen Aufgaben, deren rechnerische Durchführung im Allgemeinen doch eher kompliziert ist. 
Mit den Vektoren $\mathbf{a}=(1,0,1), \mathbf{b}=(0,1,1), \mathbf{c}=(0,2,0,4,0,9)$ und $\mathbf{r}=$ $\left(r_{1}, r_{2}, r_{3}\right)$ kann das Gleichungssystem (2) in der einfacheren (vektoriellen) Form

$$
\left(\begin{array}{l}
1 \\
0 \\
1
\end{array}\right) \cdot x+\left(\begin{array}{l}
0 \\
1 \\
1
\end{array}\right) \cdot y-\left(\begin{array}{l}
0,2 \\
0,4 \\
0,9
\end{array}\right)=\left(\begin{array}{l}
r_{1} \\
r_{2} \\
r_{3}
\end{array}\right) \Rightarrow \mathbf{a} \cdot x+\mathbf{b} \cdot y-\mathbf{c}=\mathbf{r}
$$

geschrieben werden, wobei $\mathbf{c}$ als Konstantenvektor und $\mathbf{r}$ als Residuenvektor bezeichnet wird. Die Gleichung (3) lässt nun eine einfache geometrische Interpretation zu:

Der Residuenvektor $\mathbf{r}$ kann als Differenz eines Vektors $\mathbf{d}=\mathbf{a} \cdot x+\mathbf{b} \cdot y$ und des Konstantenvektors $\mathbf{c}$ aufgefasst werden, wobei der Vektor $\mathbf{d}$ als Linearkombination der Vektoren $\mathbf{a}$ und $\mathbf{b}$ geschrieben werden kann. Machen wir die Vektoren im Ursprung fest, so liegt der Vektor $\mathbf{d}$ in der von $\mathbf{a}$ und $\mathbf{b}$ aufgespannten Ebene.

In Abbildung 2 haben wir diese Situation skizziert:

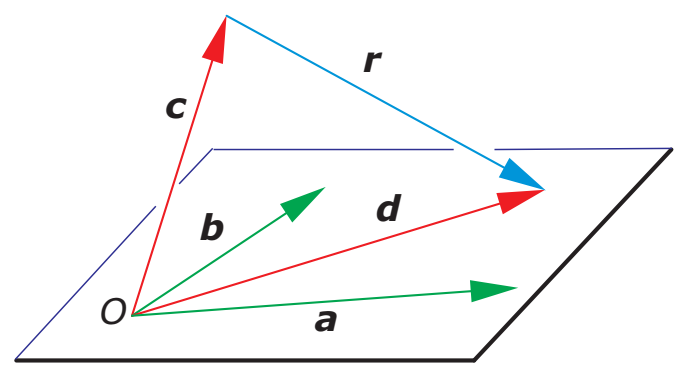

Abbildung 2

Nun ist es aber geometrisch nahe liegend, diejenige Lösung als optimale Lösung unseres Problems anzusehen, für die der Residuenvektor $\mathbf{r}$ minimale Länge hat. Die (euklidische) Länge des Vektors $\mathbf{r}$ ist aber genau dann minimal, wenn der Vektor $\mathbf{r}$ zu der von $\mathbf{a}$ und $\mathbf{b}$ aufgespannten Ebene normal steht.

\section{Erste geometrische Lösung}

Wir haben damit die in der Ebene gegebene Aufgabe (durch „räumliche Deutung“) übergeführt in das darstellend geometrische Problem „Normale aus einem Punkt $C$ auf eine Ebene $O A B$, Durchstoßpunkt $L$ einer Geraden mit einer Ebene". 
Wir wählen als Zeicheneinheit $10 \mathrm{~cm}$, tragen die Punkte $A(1,0,1), B(0,1,1)$ und $C(0,2,0,4,0,9)$ ein (vgl. Abbildung 3), konstruieren die zu $O A B$ normale Gerade $n$ und bestimmen den Durchstoßpunkt $L$ von $n$ mit der Ebene $O A B$.

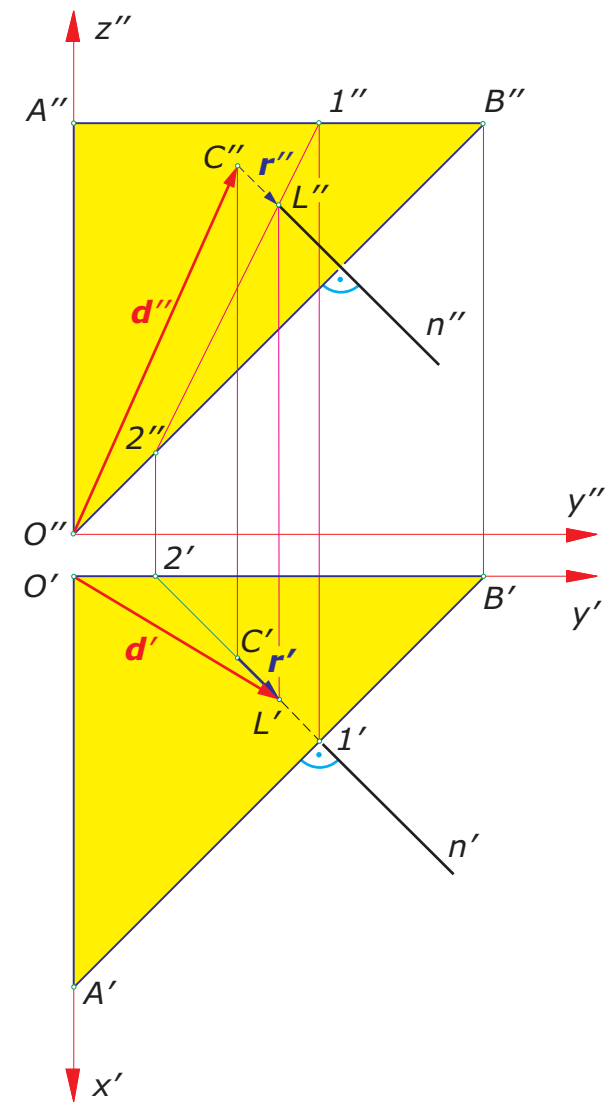

Abbildung 3

Aus der Zeichnung können wir die Länge des Residuenvektors r (als Maß für die Güte des Fehlerausgleichs) und den gesuchten Lösungsvektor $\mathbf{d}$ ablesen. Auf Grund der speziellen Werte können im Grundriss die Koordinaten $x$ und $y$ des Vektors $\mathbf{d}$ bezüglich der Basisvektoren $\mathbf{a}$ und $\mathbf{b}$ (hier orthogonal und gleich lang) abgemessen werden, womit die Lösung unseres Problems mit $x_{0}=0,3$ und $y_{0}=0,5$ vorliegt. 
Im Sinne der Methode der kleinsten Summe der Abstandsquadrate ist diese Lösung optimal, da die (euklidische) Länge von $\mathbf{r}=\sqrt{x_{1}^{2}+x_{2}^{2}} \leq 1$ nach dem Satz von Pythagoras und damit auch der Wert $\left(r_{1}^{2}+r_{2}^{2}+r_{3}^{2}\right)$ minimal ist.

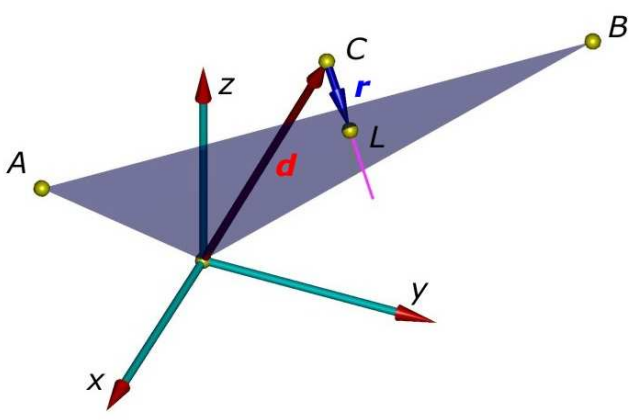

Abbildung 4

Wird die Aufgabe mit adäquater CAD-Software im Raum gelöst (Abbildung 4 ), so wird die zur Ebene $O A B$ normale Gerade $n$ mithilfe eines geeigneten Benutzerkoordinatensystems konstruiert. Da die Raumsituation dann in Echtzeit von verschiedenen Positionen aus betrachtet werden kann, ist die Einsicht in das zugrunde liegende Problem wesentlich leichter.

Bevor wir die beiden anderen Lösungsansätze (für minimale Residuen) angehen, wollen wir obiges Beispiel noch rechnerisch lösen.

\section{Numerische Lösung}

Ein möglicher Weg zur Berechnung der Werte $x_{0}$ und $y_{0}$ besteht darin, den Konstruktionsgang (Schnitt einer Geraden mit einer Ebene, Messen von Koordinaten bezüglich einer Basis) nachzurechnen. Ein wenig eleganter ist folgender Ansatz:

$\mathbf{r}$ steht zur von $\mathbf{a}$ und $\mathbf{b}$ aufgespannten Ebene genau dann normal, wenn gilt:

$\mathbf{r}$ normal $\mathbf{a}$, also $\mathbf{r} \cdot \mathbf{a}=0$ (skalares Produkt)

$\mathbf{r}$ normal $\mathbf{b}$, also $\mathbf{r} \cdot \mathbf{b}=0$ (skalares Produkt) 
Wenden wir diese Idee auf unsere Aufgabe an,

$$
0=\mathbf{r} \cdot \mathbf{a}=\left[\left(\begin{array}{l}
1 \\
0 \\
1
\end{array}\right) \cdot x+\left(\begin{array}{l}
0 \\
1 \\
1
\end{array}\right) \cdot y-\left(\begin{array}{l}
0,2 \\
0,4 \\
0,9
\end{array}\right)\right] \cdot\left(\begin{array}{l}
1 \\
0 \\
1
\end{array}\right)
$$

bzw.

$$
0=\mathbf{r} \cdot \mathbf{b}=\left[\left(\begin{array}{l}
1 \\
0 \\
1
\end{array}\right) \cdot x+\left(\begin{array}{l}
0 \\
1 \\
1
\end{array}\right) \cdot y-\left(\begin{array}{l}
0,2 \\
0,4 \\
0,9
\end{array}\right)\right] \cdot\left(\begin{array}{l}
0 \\
1 \\
1
\end{array}\right)
$$

so erhalten wir das folgende lineare Gleichungssystem in den Variablen $x$ und $y$ :

$$
\begin{aligned}
& 2 x+y=1,1 \\
& x+2 y=1,3
\end{aligned}
$$

woraus wir mit dem Gauß'schen Eliminationsverfahren rasch die Lösungen $x_{0}=$ 0,3 und $y_{0}=0,5$ finden.

\section{Weitere Aufgaben}

a) Von einer linearen Funktion $y=f(x)=a x+b$ kennt man drei Werte, wobei die zu (exakten) $x$-Werten gemessenen Funktionswerte $f(x)$ mit Messfehlern behaftet sind.

Bestimme eine lineare Funktion so, dass die Summe der Fehlerquadrate in $y$-Richtung minimal wird (Ausgleichsproblem bei Messreihen).

\begin{tabular}{c|c|c|c}
$x$ & 0 & 1 & 2 \\
\hline$f(x)$ & 2 & 1,2 & 1,6
\end{tabular}

b) In der $(x, y)$-Ebene sind drei Punkte $P_{i}\left(x_{i}, y_{i}\right)$ gegeben. Es soll eine Gerade $g: y=a x+b$ bestimmt werden, die möglichst nahe bei $P_{i}$ liegt. Zeichne die Geraden $g$ ein.

1) $P_{1}(0,5,0,5), P_{2}(2,1,5), P_{3}(2,5,1)$

2) $P_{1}(1,1), P_{2}(2,2), P_{3}(3,2)$ 


\section{Lösungsansätze für die Aufgaben}

Mit Hilfe der Fehlergleichungen

$$
\begin{aligned}
& a \cdot x_{i}+b-y_{i}=r_{i} \\
& a \cdot 0+b-2=r_{1} \\
& a \cdot 1+b-1,2=r_{2} \\
& a \cdot 2+b-1,6=r_{3}
\end{aligned} \quad \Rightarrow \quad\left(\begin{array}{l}
0 \\
1 \\
2
\end{array}\right) \cdot a+\left(\begin{array}{l}
1 \\
1 \\
1
\end{array}\right) \cdot b-\left(\begin{array}{l}
2,0 \\
1,2 \\
1,6
\end{array}\right)=\left(\begin{array}{l}
r_{1} \\
r_{2} \\
r_{3}
\end{array}\right)
$$

finden wir die Vektoren $\mathbf{f}=(0,1,2)$ (bestehend aus allen $x$-Werten der Angabepunkte), $\mathbf{e}=(1,1,1), \mathbf{c}=(2,0,1,2,1,5)$ (bestehend aus den $y$-Werten der Angabepunkte) und den gesuchten minimalen Residuenvektor $\mathbf{r}$.

Die graphische Durchführung (Zeicheneinheit $5 \mathrm{~cm}$ ) und die rechnerische Lösung führen beide auf die Gerade $g: y=-0,2 x+1,8$.

Wir haben bisher die vorhandenen Messfehler insofern korrigiert, dass die Summe der Quadrate der auftretenden Fehler minimal wird. Nun sollen diese Messfehler nach anderen Methoden minimiert werden.

\section{Methode des kleinsten maximalen Fehlers}

In unserem Beispiel wollen wir nun Werte für die Längen $x$ und $y$ ermitteln, so dass der größte auftretende Messfehler minimal wird. Geometrisch interpretiert bedeutet dies, jenen Punkt $L$ der Ebene $O A B$ zu finden, für den gilt: der Betrag der größten auftretenden Koordinatendifferenz $\left|x_{L}-x_{C}\right|,\left|y_{L}-y_{C}\right|$ und $\left|z_{L}-z_{C}\right|$, muss minimal sein.

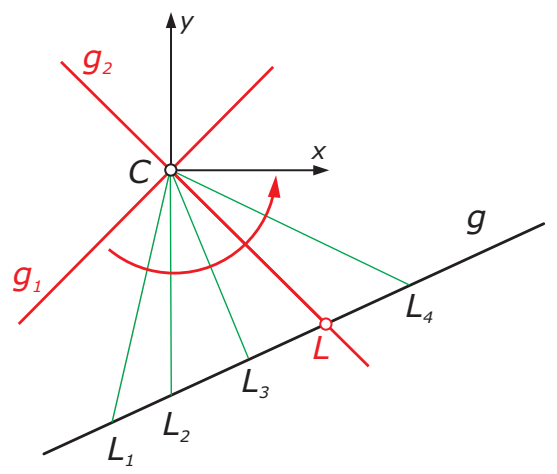

Abbildung 5 
Bemerkung. Mathematisch gesehen führt uns dies auf die Fragestellung, wann der Residuenvektor $\mathbf{r}$ im Sinne der Maximumnorm $\|\mathbf{x}\|:=\max \left(\left|x_{1}\right|,\left|x_{2}\right|,\left|x_{3}\right|\right)$ minimal wird; dies wäre die Ausgleichung nach Tschebyscheff.

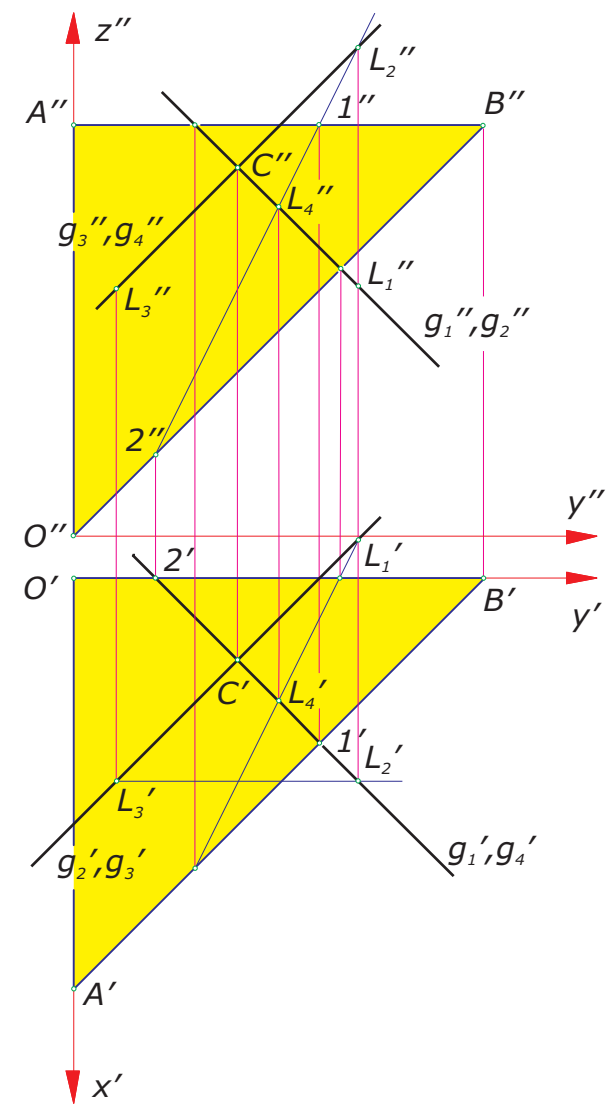

Abbildung 6

Bevor wir die Aufgabe im Raum lösen, überlegen wir uns die analoge Situation in der Ebene: Unter den Vektoren $\overrightarrow{C L_{i}}$ suchen wir jenen heraus, für den die größte auftretende Koordinatendifferenz $\left|x_{L}-x_{C}\right|,\left|y_{L}-y_{C}\right|$ am kleinsten wird. Legen wir (Abbildung 5) das zugrunde liegende Koordinatensystem in den Punkt $C$, so erkennen wir, dass $\left|x_{L}\right|=\left|y_{L}\right|$ gelten muss. Dies ist offensichtlich nur für jene Vektoren der Fall, welche die Richtungen $(1,1),(-1,1),(1,-1)$ oder 
$(-1,-1)$ aufweisen. Die gesuchten Punkte $L$ auf $g$ finden wir daher auf den durch $C$ verlaufenden Geraden $g_{1}$ und $g_{2}$ mit den Richtungsvektoren $(1,1)$ bzw. $(1,-1)$.

Übertragen wir unsere Erkenntnis in den Raum, so haben wir die Ebene $O A B$ mit den durch $C$ verlaufenden Geraden mit Richtungsvektoren $(1,1,1),(1,-1,1)$, $(1,1,-1)$ und $(1,-1,-1)$ zu schneiden und den kürzesten Vektor $\overrightarrow{C L}$ zu finden. Wir wenden zur Lösung (Abbildung 6) des Problems dieselben Grundkonstruktionen wie in Abschnitt 3 an.

Der Schnittpunkt $L_{1}(-0,1,0,7,0,6)$ mit negativer $x$-Koordinate fällt als mögliche Lösung aus.

Die Punkte $L_{2}(0,5,0,7,1,2)$ und $L_{3}(0,5,0,1,0,6)$ führen auf Lösungen, bei denen der Betrag des Maximums der Messfehler jeweils 0,3 beträgt.

Der Punkt $L_{4}(0,3,0,5,0,8)$ führt zu einem maximalen Messfehler von 0,1 und liefert die gesuchten Lösungen $x_{0}=0,3$ und $y_{0}=0,5$.

Bemerkung. Für Ausgleichsrechnungen der vorgestellten Art sind die Lösungen für die euklidische und die Maximumsnorm immer gleich, da die Normale $n$ zu Ebene $O A B$ immer die Richtung $(1,1,-1)$ aufweist; erst bei anderen Anwendungen treten unterschiedliche Ergebnisse auf.

Auch in diesem Fall gibt die Lösung (Abbildung 7) mithilfe von CAD-Software einen besseren Einblick in die Raumsituation:

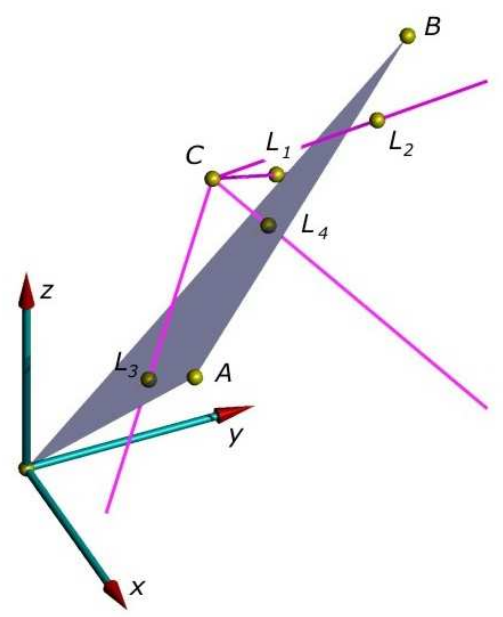

Abbildung 7 
Zur numerischen Lösung der Ausgleichung nach Tschebyscheff rechnen wir am besten den Konstruktionsgang nach:

Wir berechnen die Durchstoßpunkte $\underline{L_{i}}$ der Geraden, bestimmen anschließend die Koordinatendifferenzen der Vektoren $\overrightarrow{C L_{i}}$ und wählen als Lösung jenen Vektor r, für den das Maximum dieser Differenzen am kleinsten ist. Werden die Werte $r_{i}$ dieses Residuenvektors in (2) eingesetzt, so ergeben sich die gesuchten Werte $x_{0}=0,3$ und $y_{0}=0,5$.

\section{Methode der kleinsten Messfehlersumme}

Wir werden nun noch Werte für die Längen $x$ und $y$ so ermitteln, dass die Summe der Beträge der auftretenden Messfehler minimal wird. Wir wollen dies wieder geometrisch interpretieren: Es gilt jenen Punkt $L$ der Ebene $O A B \mathrm{zu}$ finden, für den

$$
\left|x_{L}-x_{C}\right|+\left|y_{L}-y_{C}\right|+\left|z_{L}-z_{C}\right|
$$

minimal wird.

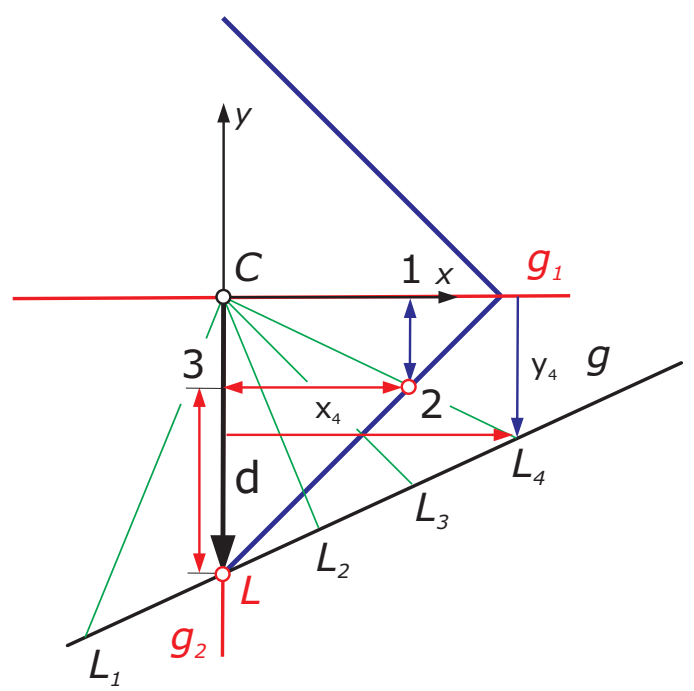

Abbildung 8 
Wiederum betrachten wir die analoge Situation in der Ebene und suchen jenen Vektor $\overrightarrow{C L_{i}}$ mit der Eigenschaft

$$
\left|x_{L}-x_{C}\right|+\left|y_{L}-y_{C}\right|
$$

ist minimal.

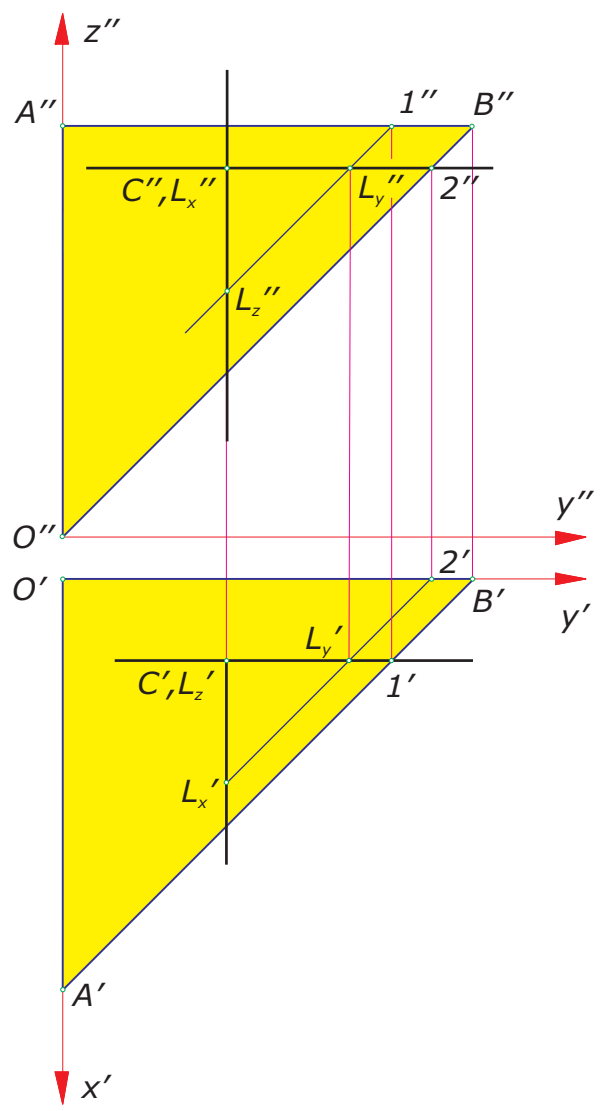

Abbildung 9

Bei angepasstem Koordinatensystem (Abbildung 8) erkennen wir, dass z.B. für den Punkt $L_{4}$ der Geraden $g: y=k . x+d(0<k<1, d<0)$ gilt:

$$
\left|x_{4}\right|+\left|y_{4}\right| \geq|\overline{12}|+|\overline{23}|=|\overline{12}|+|\overline{3 L}|=|d| .
$$


Für alle Punkte der Geraden $g$ aus Abbildung 8 gilt daher:

$$
\left|x_{i}\right|+\left|y_{i}\right| \geq|d|
$$

d.h. der Vektor $\overrightarrow{C L}=(0,-d)$ ist der gesuchte Vektor.

Betrachten wir alle anderen möglichen Lagen der Geraden $g$ zum Koordinatensystem $(k<0, k>1, d>0)$, so sehen wir, dass die Lösungen unter jenen Vektoren zu suchen sind, deren Richtungen parallel zu den Koordinatenachsen verlaufen.

Bei der Lösung (Abbildung 9) dieser Aufgabe (Zeicheneinheit $10 \mathrm{~cm}$ ) müssen wir die Ebene $O A B$ mit den durch $C$ verlaufenden achsenparallelen Geraden schneiden und den kürzesten Vektor $\overrightarrow{C L_{i}}$ bestimmen („Durchstoßpunkt einer projizierenden Geraden mit einer Ebene - Angittern“).

\section{Ein tieferer Einblick in die Materie}

a) Normen und Einheitssphären:

Vom mathematischen Standpunkt aus handelt es sich bei allen drei Lösungsansätzen um das gleiche Problem, nämlich den kürzesten Vektor im Sinne einer gewissen Norm zu finden.

Bemerkung. Die verwendeten Normen, die Euklidische Norm (Gauss), die Maximumsnorm (Tschebyscheff) und die $L_{1}$-Norm erfüllen alle folgende Eigenschaften:

1) Für jeden Vektor $\mathbf{v}$ gilt: $\|\mathbf{v}\| \geq 0$, falls $\|\mathbf{v}\|=0 \Rightarrow \mathbf{v}=\mathbf{o}$

2) Für jeden Vektor $\mathbf{v}$ gilt: $\|a \cdot \mathbf{v}\|=|a| \cdot\|\mathbf{v}\|$

3) $\|\mathbf{x}+\mathbf{y}\| \leq\|\mathbf{x}\|+\|\mathbf{y}\|$ (Dreiecksungleichung),

was auch mit elementaren mathematischen Mitteln leicht nachvollziehbar ist.

Um ein gewisses Gefühl für die drei verwendeten Normen zu erhalten und unsere geometrischen Konstruktionen aus einem anderen Blickwinkel zu sehen, betrachten wir die „Einheitsscheibe“ in der Ebene

$$
\left\{\mathbf{x} \in R^{2} \text { mit }\|\mathbf{x}\| \leq 1\right\}
$$

bzw. den „Einheitsball“ im Raum

$$
\left\{\mathbf{x} \in R^{3} \text { mit }\|\mathbf{x}\| \leq 1\right\} .
$$

Für die einzelnen Normen ergibt dies folgende Strukturen: 
Euklidische Norm:

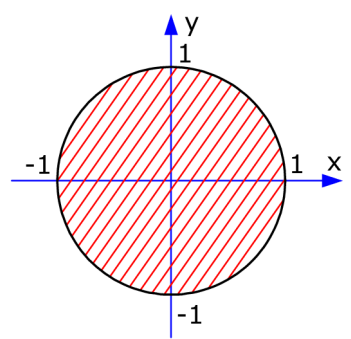

$\sqrt{x_{1}^{2}+x_{2}^{2}} \leq 1$

im $R^{2} \quad$ „Kreisscheibe“ $\operatorname{im} R^{3} \quad$ „Ball“

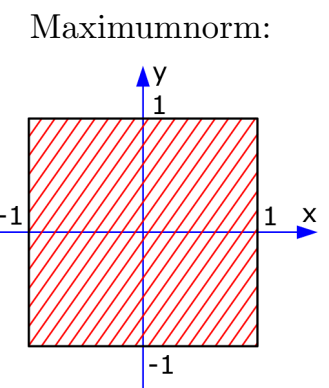

$\max \left(\left|x_{1}\right|,\left|x_{2}\right|\right) \leq 1$

„achsenparalleles Quadrat" „Würfel in Standardaufstellung“"
$L_{1}$-Norm:

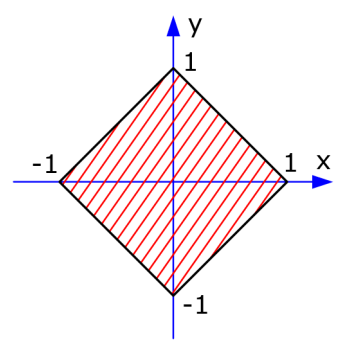

$\left|x_{1}\right|+\left|x_{2}\right| \leq 1$

"verdrehtes Quadrat"

„Oktaeder"

b) Messen mittels Einheitssphären: Eine geometrische Interpretation der Abstandsbestimmung zwischen zwei Punkten $A$ und $B$ besteht nun darin, den Einheitskreis mit Mitte $A$ so lange aufzublasen (zentrische Ähnlichkeit), bis der Punkt $B$ auf der Oberfläche (Umfang) des Einheitskreises zu liegen kommt. Der Radius des so erhaltenen Einheitskreises ist die Länge der gesuchten Strecke (im Sinne der verwendeten Metrik).

Zur Bestimmung des Abstandes eines Punktes $C$ von einer Ebene $O A B$ müssen wir also die Einheitssphäre um den Mittelpunkt $C$ so lange ,,aufblasen", bis diese die Ebene in einem Punkt $L$ berührt. Die Gerade $C L$ trägt dann den minimalen Abstand zwischen der Ebene und dem Punkt.

Bei der Verwendung der euklidischen Norm liegt der Berührpunkt $L$ der Messsphäre im (euklidischen) Normalfußpunkt; es gibt immer eine eindeutige Lösung.

Liegt die Maximumsnorm oder die $L_{1}$-Norm der Messung zugrunde, so können auch mehr als eine Lösung auftreten:

die Einheitssphäre (hier ein Würfel bzw. ein Oktaeder) berührt die Ebene längs einer Geraden $\rightarrow$ einparametrige Schar von Lösungen

die Ebene ist zu einer Seitenfläche der Einheitssphäre parallel $\rightarrow$ zweiparametrige Schar von Lösungen

c) Verallgemeinerung: Im Geometrieunterricht können wir nur die dreidimensionalen Aufgaben, also die Lösung der Ausgleichsrechnung für drei (voneinander abhängige) Messungen konstruktiv ausführen. Allgemein kann das Problem für $n$ mit Fehlern behafteten Messungen die Ausgleichsrechnung 
durchzuführen auf die Bestimmung des kürzesten Abstandes zwischen einem Punkt $C$ und einer Hyperebene zurückgeführt werden.

\section{Literatur}

[1] K. Nipp and D. Stoffer, Lineare Algebra, Verlag der Fachvereine Zürich, Zürich, 1992.

[2] U. Wolf, Konstruktion der Ausgleichsgeraden dreier Punkte, Der Mathematikunterricht 2/82, 57-65, Ernst Klett Verlag, Stuttgart.

ANDREAS ASPERL

INSTITUT FÜR DISKRETE MATHEMATIK UND GEOMETRIE

TU WIEN

WIEDNER HAUPTSTR. 8-10

A-1040 WIEN

AUSTRIA

E-mail: aa@geometrie.tuwien.ac.at

(Received October, 2004) 